\title{
Characteristics of and Virulence Factors Associated with Biofilm Formation in Clinical Enterococcus faecalis Isolates in China
}

\begin{abstract}
Jin-Xin Zheng ${ }^{1 t}$, Yang Wu ${ }^{21}$, Zhi-Wei Lin ${ }^{1 t}$, Zhang-Ya Pu't, Wei-Ming Yao', Zhong Chen', Duo-Yun Li', Qi-Wen Deng ${ }^{1}$, Di Qu ${ }^{2 *}$ and Zhi-Jian Yu${ }^{1 *}$
\end{abstract}

OPEN ACCESS

Edited by:

Awdhesh Kalia,

University of Texas MD Anderson Cancer Center, United States

Reviewed by:

Pallab Ghosh,

Harvard Medical School,

United States

J. Christopher Fenno,

University of Michigan, United States

*Correspondence:

Di Qu

dqu@fudan.edu.cn

Zhi-Jian Yu

yuzhijiansmu@163.com

${ }^{\dagger}$ These authors have contributed equally to this work.

Specialty section: This article was submitted to Infectious Diseases,

a section of the journal

Frontiers in Microbiology

Received: 06 April 2017 Accepted: 13 November 2017 Published: 24 November 2017

Citation:

Zheng J-X, Wu Y, Lin Z-W, Pu Z-Y,

Yao W-M, Chen Z, Li D-Y,

Deng Q-W, Qu D and Yu Z-J (2017)

Characteristics of and Virulence

Factors Associated with Biofilm Formation in Clinical Enterococcus faecalis Isolates in China. Front. Microbiol. 8:2338. doi: 10.3389/fmicb.2017.02338
' Department of Infectious Diseases and Shenzhen Key Laboratory for Endogenous Infection, Shenzhen Nanshan People's Hospital of Shenzhen University, Shenzhen, China, ${ }^{2}$ Key Laboratory of Medical Molecular Virology of Ministries of Education and Health, School of Basic Medical Science and Institutes of Biomedical Sciences, Shanghai Medical College of Fudan University, Shanghai, China

Enterococcus faecalis biofilm traits and distribution characteristics in China have not been clarified. This study aimed to determine the prevalence and characteristics of $E$. faecalis biofilm formation in a sample of clinical isolates and to explore the virulence factors associated with biofilm formation in those isolates. A total of 265 E. faecalis isolates were collected from patients in Shenzhen, China. Virulence genes were detected within the genomes of the microbes by polymerase chain reaction. The isolates were subjected to multilocus sequence typing (MLST) based on housekeeping genes. Biofilms were detected by crystal violet staining. The expression levels of the clinical $E$. faecalis isolates' genes were determined by quantitative real-time polymerase chain reaction. The prevalence of biofilm formation among $E$. faecalis clinical isolates was $47.2 \%$. MLST yielded 44 different sequence types (STs). The main STs were ST16 and ST179; the ST16 isolates were more likely to form strong or medium biofilm than the ST179 isolates $(p<0.001)$. Strong or medium biofilm formation was more common in linezolid-resistant isolates than in linezolid-sensitive isolates $(p=0.001)$. Biofilm formation was more frequently detected in enterococcal surface protein (esp+), surface aggregating protein (asa1+), cytolysin $\mathrm{A}(\mathrm{cy} / \mathrm{A}+)$, or aggregation substance $(\mathrm{agg}+)$ positive isolates than in isolates that were negative $(-)$ for these virulence factors. Multivariate regression analysis indicated that $c y / A$ [odds ratio $(\mathrm{OR}) 4.083, p<0.001$ ] was a risk factor for weak biofilm formation, and that esp (OR 8.207, $p<0.001$ ) was a risk factor for strong or medium biofilm formation. The expression of $c y / A$ was raised (4.02 to 6.00 -fold) in weak biofilm isolates compared to the biofilm-negative isolates, and the expression of esp was greatly elevated (11.39 to 134.08-fold) in strong biofilm isolates compared to biofilm-negative isolates. In conclusion, the ST16 classification and linezolid resistance were positively associated with strong/medium biofilm formation in clinical E. faecalis isolates. cylA was associated with weak biofilm formation, and esp was only associated with strong or medium biofilm formation of the clinical $E$. faecalis isolates.

Keywords: Enterococcus faecalis, biofilm formation, virulence genes, multilocus sequence typing, linezolid resistance 


\section{INTRODUCTION}

Enterococci are normal gut microbes in humans and other animals (Sghir et al., 2000; Damborg et al., 2009). However, in the last two decades, enterococcus pathogens have emerged as a major cause of nosocomial infections affecting various tissues, including the urinary tract, respiratory tract, peritoneum, and bloodstream (Treitman et al., 2005; Bonten and Willems, 2012). The most prevalent species cultured from humans, Enterococcus faecalis and Enterococcus faecium, account for more than 90\% of clinical enterococcal isolates (Fisher and Phillips, 2009). It is noteworthy that E. faecalis and E. faecium cause treatment to be increasing difficult because of their intrinsic and acquired resistance to many antibiotics. E. faecalis and E. faecium have resistance to many commonly used antimicrobial agents, such as ampicillin and vancomycin (Van Harten et al., 2017). VRE especially have emerged as a major cause of outbreaks of nosocomial infection, which with their extensive resistance to a plethora of antibiotics have attracted more and more attention in recent years (Flokas et al., 2017). Linezolid, as the first antimicrobial agent of the oxazolidinones class, was widely used to treat VRE infections. Unfortunately, growing cases of linezolid-resistant enterococci have emerged in hospitals (Gawryszewska et al., 2017).

In addition to drug resistance, enterococci usually with a high capacity of biofilm formation, which has increased the difficulty of treatment. In Italy, $86.7 \%$ of E. faecalis clinical isolates formed different levels of biofilms, whereas only $15.6 \%$ of E. faecium isolates formed biofilms (Dupre et al., 2003). Among 109 enterococcal bloodstream isolates from Britain, $100 \%$ of E. faecalis strains had the ability to form biofilm, but only $42 \%$ of E. faecium isolates formed biofilms (Sandoe et al., 2003). E. faecalis isolates from the USA also had a high capacity of biofilm formation, Mohamed et al. found 93\% of 163 E. faecalis isolates from various sources classified as biofilm producers (Mohamed et al., 2004). Another study from Japan indicated $100 \%$ of $352 \mathrm{E}$. faecalis isolates in urinary tract infections with different levels of biofilm formation (Seno et al., 2005). However, only $57.2 \%$ of $E$. faecalis isolates from Spain had the ability to form biofilms, and the $39 \mathrm{E}$. faecium isolates in the study couldn't produce biofilms (Toledo-Arana et al., 2001). These studies found the E. faecalis isolates to have a higher capacity of biofilm formation than the E. faecium isolates, and the prevalence of $E$. faecalis biofilm differs regionally. However, up to now, the prevalence and characteristics of $E$. faecalis biofilm in China have remained obscure.

The phylogenetic relationships of pathogenic bacteria, including Staphylococcus spp. and Enterococcus spp., have been examined with MLST (Urwin and Maiden, 2003), a methodology that has yielded STs with distinctive virulence and drug resistance strain profiles (Manning et al., 2009). Biofilm formation has also

Abbreviations: Agg, aggregation substance; Asa1, surface aggregating protein; CI, confidence intervals; CLSI, Clinical and Laboratory Standards Institute; CylA, cytolysin A; eDNA, extracellular DNA; Esp, enterococcal surface protein; GelE, gelatinase; MIC, minimal inhibitory concentration; MLST, multilocus sequence typing; OR, Odds ratio; qRT-PCR, quantitative real-time polymerase chain reaction; ST, sequence type; VRE, vancomycin-resistant enterococci. been shown to vary across isolate STs (Kozitskaya et al., 2005). ST27 of Staphylococcus epidermidis, which is prone to biofilm formation, was found to occur preferentially in hospitals and to differ from clones in the community (Kozitskaya et al., 2005). The ST17 and ST19 lineages of group B Streptococcus strains isolated from invasive disease case samples were found to form weak biofilms relative to strains recovered from individuals with asymptomatic colonization (Parker et al., 2016). The biofilm formation properties of different STs of E. faecalis have not been characterized.

Previous researchers have reported that some virulence factors play important roles in the pathogenicity of E. faecalis, and several virulence factors may be related to $E$. faecalis biofilm formation. Esp is a large surface protein, which has been found to support cell adherence, colonization, and persistence in the urinary tract, evasion of the immune system, and to play an important role in E. faecalis biofilm formation (Toledo-Arana et al., 2001; Paganelli et al., 2012). GelE is an extracellular metalloprotease that hydrolyzes gelatin, collagen, and hemoglobin. GelE was responsible for regulating autolysis and the release of highmolecular-weight eDNA, an important component for the development of E. faecalis biofilms, and has also been reported to contribute to the bacterial adherence and biofilm formation of E. faecalis (Kayaoglu and Ørstavik, 2004; Park et al., 2007; Thomas et al., 2008). However, some research groups have found no significant correlation between the presence of Esp or GelE and E. faecalis biofilm formation (Kristich et al., 2004; Mohamed and Murray, 2005; Anderson et al., 2016).

Another virulence factor of E. faecalis, the Asal, mediates aggregation between bacteria and enables the transfer of plasmids, was also found to promote biofilm formation in one study (Süßmuth et al., 2000; Chuang-Smith et al., 2010). The cylA gene of $E$. faecalis synthesizes a protein involved in the activation of cytolysin, and the lytic action of cytolysin on various cell types has been explored, including its contribution to the virulence of E. faecalis in infections (Van Tyne et al., 2013). Another study found cylA may be associated with E. faecalis biofilm formation in urinary tract infections (Seno et al., 2005). However, evidence of the involvement of asa 1 and cylA in E. faecalis biofilm formation is still deficient and needs to be confirmed by further studies. Hence, the virulence factors that enable $E$. faecalis biofilm formation remain ambiguous and controversial.

The aim of the present study was to explore the prevalence and characteristics of E. faecalis biofilm, and to identify virulence factors associated with E. faecalis biofilm formation. To our knowledge, this is the first study to combine data on biofilm formation, virulence genes, antibiotic resistance, and MLST of E. faecalis from China.

\section{MATERIALS AND METHODS}

\section{Bacterial Isolates}

A total of 265 clinical E. faecalis isolates were obtained from patients at Shenzhen Nanshan Hospital, Shenzhen University in China between January 12010 and June 30 2016. Information on the antibiotics used in each case was collected from the electronic 
medical records database. Antibiotic use was as follows: 44 cases involved penicillins (ampicillin, piperacillin, amoxicillin), 15 cases involved glycopeptides (vancomycin, teicoplanin), 4 cases involved linezolid, and 37 cases involved other antibiotics (ciprofloxacin, levofloxacin, moxifloxacin, minocycline). The strains isolated from the patients were identified with a VITEK 2 microbial testing system (bioMérieux, Marcy l'Etoile, France). E. faecalis ATCC29212 and OG1RF (ATCC47077) strains purchased from the American Type Culture Collection were used as reference strains. All procedures involving human participants were performed in accordance with the ethical standards of Shenzhen University and with the 1964 Helsinki declaration and its later amendments, or comparable ethical standards. For this type of study, formal consent is not required.

\section{Antibiotic-Susceptibility Testing}

The susceptibilities of the isolates to clinically relevant antibiotics (i.e., penicillin G, ampicillin, imipenem, ciprofloxacin, levofloxacin, teicoplanin, vancomycin, tigecycline, cotrimoxazole, linezolid, high-level gentamicin, erythromycin, and tetracycline) were determined with the VITEK 2 system (bioMérieux, Marcy l'Etoile, France). We determined the MICs of ampicillin, vancomycin, and linezolid using the broth macrodilution method according to CLSI guidelines, with CLSI-recommended MIC breakpoints.

\section{Isolation of DNA}

DNA was extracted from isolates and purified with a DNeasy Blood and Tissue Kit (Qiagen China Co., Ltd., Zhangjiang Hi-Tech Park Pudong, Shanghai, China) according to the manufacturer's protocol for Gram-positive bacteria. The microbial DNA was eluted with $100 \mu \mathrm{l}$ of AE buffer (Qiagen) and stored at $-20^{\circ} \mathrm{C}$.

\section{Detection of Virulence Genes by PCR}

The extracted DNA served as a template for the amplification of virulence genes. All primer sequences and corresponding references are listed in Table 1 (Vankerckhoven et al., 2004; Dupre et al., 2003). PCR amplification was performed in a total volume of $50 \mu \mathrm{l}$, containing $2 \times$ PCR Master Mix (Tiangen Biotech Beijing Co., Ltd., Beijing, China), $0.5 \mathrm{mM}$ of each primer, and $1 \mu \mathrm{l}$ template DNA. The cycling conditions were as follows: $95^{\circ} \mathrm{C}$ for $3 \mathrm{~min}$; followed by 30 cycles at $95^{\circ} \mathrm{C}$ for $30 \mathrm{~s}, 52^{\circ} \mathrm{C}$ for $30 \mathrm{~s}, 72^{\circ} \mathrm{C}$ for $60 \mathrm{~s}$; and a final $10 \mathrm{~min}$ extension step at $72^{\circ} \mathrm{C}$. Each PCR set included a no-template control and a positive control. The amplification products were analyzed by electrophoresis in $1.0 \%$ agarose gels.

\section{Multilocus Sequence Typing}

Multilocus sequence typing was conducted by the reference method (Ruiz-Garbajosa et al., 2006). Briefly, seven housekeeping genes ( $g d h, g y d, p s t S, g k i, a r o E$, $x p t$, and $y q i L)$ were PCR amplified and sequenced. All primer sequences and corresponding references are listed in Table 2 . The PCR system and cycling conditions were the same as indicated above for the virulence genes. For each locus, a distinct allele number was assigned to each unique sequence, in accordance with the E. faecalis MLST database $^{1}$. The seven STs were assigned in the order $g d h, g y d, p s t S$, $g k i$, aroE, $x p t$, and $y q i L$, corresponding to the allele numbers at the seven loci. ST names were kept consistent as much as possible for the same strains.

\section{Biofilm Assay}

Enterococcus faecalis biofilm formation was detected according to the reference method, with some modifications (Toledo-Arana et al., 2001; Mohamed et al., 2004). Briefly, bacteria that had been grown overnight were diluted 1:100 in $200 \mu$ l of tryptic soy broth with $0.25 \%$ glucose and inoculated onto polystyrene microtiter plates (Costar3599; Corning). After $24 \mathrm{~h}$ of static incubation at $37^{\circ} \mathrm{C}$, the supernatant was discarded, and plates were washed thrice with deionized water to remove unattached cells, then fixed with methanol for $30 \mathrm{~min}$, stained with $1 \%$ crystal violet for $30 \mathrm{~min}$, and rinsed with distilled water. The optical density at $570 \mathrm{~nm}\left(\mathrm{OD}_{570}\right)$ was determined. Each assay was performed in triplicate on at least three occasions.

\section{RNA Extraction and qRT-PCR}

The expression levels of the esp and $c y l A$ genes of E. faecalis clinical isolates were determined by qRT-PCR. The primers used for qRT-PCR are listed in Table 3. The RNA extraction was carried out according to the reference method (Xu et al., 2017), with some modifications: the E. faecalis strains were cultured at $37^{\circ} \mathrm{C}$ until the $\mathrm{OD}_{600}$ reached 0.6 . Then, the cell pellets were washed with ice-cold normal saline and then homogenized using $0.1 \mathrm{~mm}$ Zirconia-silica beads in a Mini-BeadBeater (Biospec, Bartlesville, OK, United States) at a speed of 4,000 rpm for $1 \mathrm{~min}$, followed by cooling on ice for $1 \mathrm{~min}$. This homogenization and cooling cycle was repeated five times; then, the samples were centrifuged at 15,000 rpm and the bacterial RNA in the supernatant was purified using an RNeasy Mini Kit (Qiagen) and quantified using an ND-1000 spectrophotometer (NanoDrop Technologies, Wilmington, DE, United States). RNA samples that had a 260/280 ratio between 2.0 and 2.2 were reverse transcribed with the PrimeScript RT Reagent Kit (TaKaRa). Finally, qRT-PCR was performed with the SYBR Premix Ex Taq II Kit (TaKaRa) on the Mastercycler ep realplex system (Eppendorf), with an initial incubation at $95^{\circ} \mathrm{C}$ for $2 \mathrm{~min}$, followed by 40 cycles of $15 \mathrm{~s}$ at $95^{\circ} \mathrm{C}$, and $60 \mathrm{~s}$ at $60^{\circ} \mathrm{C}$. Each sample was analyzed in triplicate.

For all samples, the internal control gene $\operatorname{rec} A$ (Ruiz-Cruz et al., 2015) was used to normalize the expression of the esp and cylA genes. The threshold cycle (Ct) numbers were confirmed by the detection system software and the data were analyzed based on the $2^{-\Delta \Delta \mathrm{Ct}}$ method.

\section{Statistical Analysis}

The prevalence of E. faecalis biofilm is reported as a number (percentage) and was compared using the chi-square test or Fisher's exact test. The virulence factors associated with biofilm formation were analyzed with a multivariate logistic regression model constructed by backward selection based on the Wald

\footnotetext{
${ }^{1}$ http://efaecalis.mlst.net/
} 
statistic. ORs are reported with 95\% CIs. $P$-values $<0.05$ were regarded as statistically significant. All data were analyzed using the statistical software SPSS (version 14.0; SPSS, Chicago, IL, United States).

\section{RESULTS}

\section{Biofilm Formation}

$\mathrm{OD}_{570}$ microplate readings after crystal violet staining ranged from 0.05 to 3.5 . The numbers and percentages of the isolates, altogether and segregated by clinical source, and the biofilm phenotype categorized based on the approach of others (ToledoArana et al., 2001; Mohamed et al., 2004), with a strong $\left(\mathrm{OD}_{570}>2\right)$, medium $\left(\mathrm{OD}_{570}, 1-2\right)$, or weak $\left(\mathrm{OD}_{570}>0.5\right.$ and $<1$ ) biofilm formation phenotype, are reported in Table 4. The median $\mathrm{OD}_{570}$ values for control strains OG1RF [weak biofilm (Montealegre et al., 2015)] and ATCC29212 were 0.56 and 0.15, respectively.

As reported in Table 4, biofilms were observed for nearly half of all of the E. faecalis isolates examined, and among these biofilm-forming strains, the majority were classified as having a weak biofilm phenotype. With respect to clinical source, isolates from urine were the most likely to exhibit biofilm formation,

TABLE 1 | PCR primers used for detection of Enterococcus faecalis virulence factors.

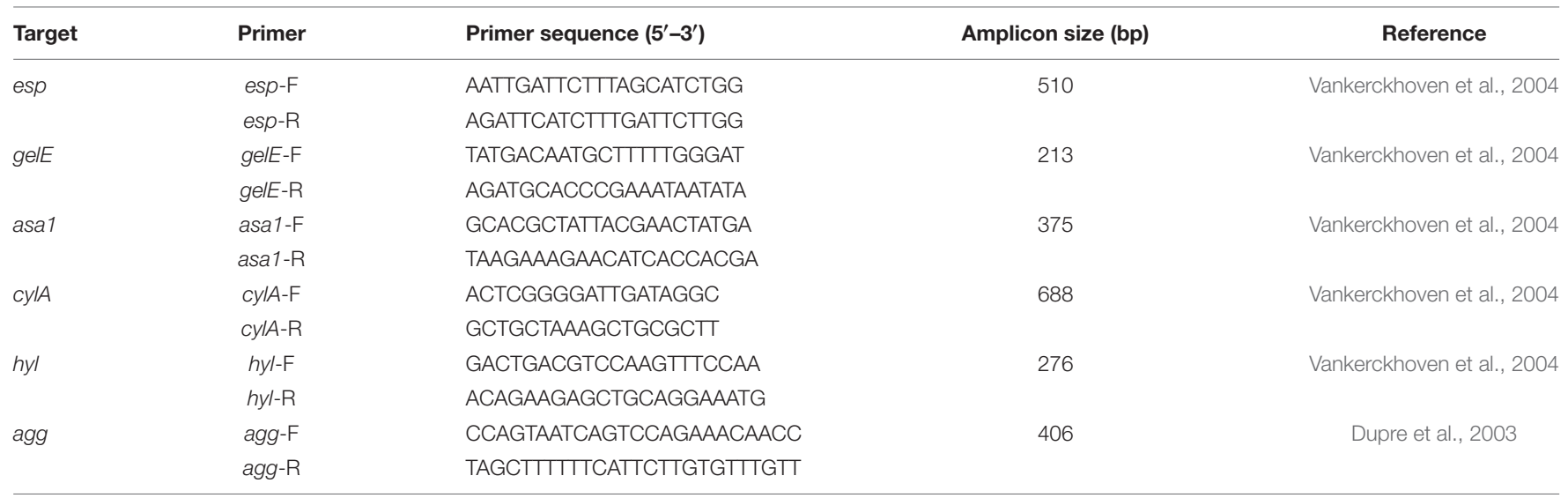

TABLE 2 | PCR primers used for E. faecalis MLST gene diversity determination.

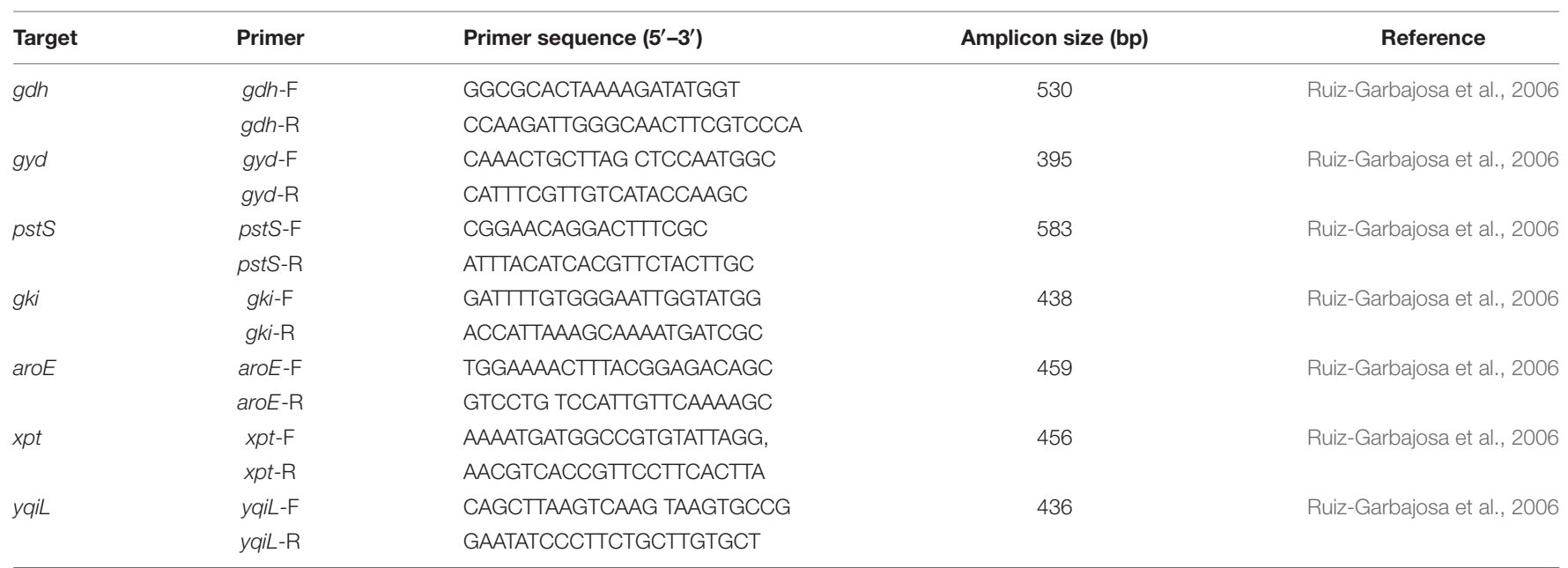

TABLE 3 | PCR primers used for determining the expression levels of esp and cylA of E. faecalis isolates by quantitative real-time polymerase chain reaction ( $q R T$-PCR).

\begin{tabular}{|c|c|c|c|c|}
\hline Target & Primer & Primer sequence $\left(5^{\prime}-3^{\prime}\right)$ & Amplicon size (bp) & Reference \\
\hline \multirow[t]{2}{*}{ recA } & recA-F & CGACTAATGTCTCAAGCACTAC & 106 & This study \\
\hline & recA-R & CGAACATCACGCCAACTT & & \\
\hline \multirow[t]{2}{*}{ esp } & Resp-F & GCATCAGTATTAGTTGGT & 196 & This study \\
\hline & Resp-R & TTCCTTGTAACACATCAC & & \\
\hline \multirow[t]{2}{*}{ CyIA } & RcylA-F & GGAGGATATGGTGACAAT & 163 & This study \\
\hline & RcylA-R & TTACTTCTGGAGTTGCTAA & & \\
\hline
\end{tabular}


TABLE 4 | Occurrence of E. faecalis biofilm formation by clinical source.

\begin{tabular}{|c|c|c|c|c|c|}
\hline \multirow[t]{2}{*}{ Clinical source (no. isolates tested) } & \multicolumn{5}{|c|}{ No. (\%) of isolates with biofilm phenotype } \\
\hline & Strong & Medium & Strong or medium & Weak & All positive \\
\hline Blood (25) & $5(20.0)$ & $1(4.0)$ & $6(24.0)$ & $4(16.0)$ & $10(40.0)$ \\
\hline Urine (113) & 15 (13.3) & $15(13.3)$ & 30 (26.6) & 27 (23.9) & $57(50.4)$ \\
\hline Pus or secretions (64) & $6(9.4)$ & $1(1.6)$ & $7(11.0)$ & $22(34.4)$ & 29 (45.3) \\
\hline Bile (19) & $1(5.3)$ & $4(21.1)$ & $5(26.3)$ & $2(10.5)$ & $7(36.8)$ \\
\hline Other ${ }^{\mathrm{a}}(44)$ & $8(18.2)$ & $3(6.8)$ & $11(25.0)$ & $11(25.0)$ & $22(50.0)$ \\
\hline Total (265) & $35(13.2)$ & $24(9.1)$ & 59 (22.3) & $66(24.9)$ & $125(47.2)$ \\
\hline
\end{tabular}

asputum, tissue, catheters, pleural effusion, ascites fluid, amniotic fluid, puncture fluid, cerebrospinal fluid, throat swabs.

followed by isolates from bile; the likelihood of biofilm formation was statistically similar across the remaining sources.

\section{Association between ST and Biofilm Formation}

Multilocus sequence typing of $265 \mathrm{E}$. faecalis clinical isolates enabled us to determine the STs for 224 isolates. We found 44 different STs, 41 of which were represented by four or fewer isolates. The biofilm characteristics and numbers of isolates assigned to each ST are reported in Supplementary Table S1.

Because ST16 and ST179 were quite dominant, accounting for $65.6 \%(147 / 224)$ of the isolates in this study, the biofilm formation characteristics of the ST16 and ST179 isolates were subjected to further analysis. As shown in Table 5, biofilm formation, and strong or medium biofilm formation in particular, were detected significantly more frequently among ST16 isolates than among ST179 isolates. Further analysis of the clinical sources of ST16 and ST179 E. faecalis isolates indicated that the characteristics of each were consistent across clinical sources (Supplementary Table S2).

\section{Association between Biofilm Formation and Linezolid Susceptibility}

Using the broth macrodilution method to determine the MICs of ampicillin, vancomycin, and linezolid, and a resistance criterion classification of MIC $\geq 8 \mu \mathrm{g} / \mathrm{ml}$, we found 10 strains with linezolid resistance (the 10 patients received no linezolid treatment), only four strains with ampicillin resistance (only 1 of the 4 patients received piperacillin-tazobactam treatment), and none with

TABLE 5 | Biofilm-forming capacity comparison between ST16 and ST179 isolates.

\begin{tabular}{|c|c|c|c|}
\hline \multirow[t]{2}{*}{ Biofilm phenotype } & \multicolumn{2}{|c|}{ No. (\%) with phenotype } & \multirow[t]{2}{*}{$P$} \\
\hline & $S T 16, N=79$ & $S T 179, N=68$ & \\
\hline Strong & $19(24.1)$ & $1(1.5)$ & $<0.001$ \\
\hline Medium & $9(11.4)$ & $3(4.4)$ & NS \\
\hline Strong or medium & $28(35.4)$ & $4(5.9)$ & $<0.001$ \\
\hline Weak & $20(25.3)$ & $24(35.3)$ & NS \\
\hline All positive & $48(60.8)$ & $28(41.2)$ & 0.018 \\
\hline
\end{tabular}

NS, not significant. vancomycin resistance. Follow-up analysis of the relationship between $E$. faecalis biofilm formation and linezolid susceptibility showed that the linezolid-resistant strains were more likely to exhibit biofilm formation than the linezolid-sensitive strains $(\mathrm{MIC} \leq 2 \mu \mathrm{g} / \mathrm{ml})$, and that the biofilm formation characteristics of the isolates with a linezolid MIC in the intermediate range $(2-8 \mu \mathrm{g} / \mathrm{ml} ; n=41)$ did not differ significantly from that of the lower or higher MIC isolate groups (Table 6). More specifically, strong or medium biofilm formation was more prevalent among linezolid-resistant isolates than among linezolid-sensitive isolates (Table 6). Two ST16 isolates and no ST179 isolates were included among the linezolid-resistant strains; biofilm prevalence did not differ significantly between linezolid-sensitive and -resistant isolates (Supplementary Table S3).

\section{Correlation between Biofilm Formation and Virulence Factors}

As reported in Table 7, the analysis of PCR-amplified virulence factors showed that the esp-positive $(+)$ E. faecalis isolates had a significantly greater prevalence of biofilm formation than the esp-negative $(-)$ isolates. Additionally, biofilm formation was detected more frequently in $a s a 1+, c y l A+$ (cytolysin A-encoding gene positive), and agg+ (aggregation substance-encoding gene positive) isolates than in isolates not expressing each of these virulence factors (Table 7). Conversely, gelE- isolates were significantly more likely to have biofilm formation than gelE+ isolates, especially biofilms categorized as strong or medium (Table 7, see Footnote).

TABLE 6 | Correlation between biofilm-forming capacity and linezolid sensitivity.

\begin{tabular}{llcc}
\hline Biofilm phenotype & \multicolumn{2}{c}{ No. (\%) with linezolid MIC in $\boldsymbol{\mu} \mathbf{g} / \mathbf{m l}$} \\
\cline { 2 - 4 } & $\begin{array}{l}\text { MIC } \leq \mathbf{2}, \\
\mathbf{N}=\mathbf{2 1 4}\end{array}$ & $\begin{array}{c}\text { MIC 2-8, } \\
\mathbf{N}=\mathbf{4 1}\end{array}$ & $\begin{array}{l}\text { MIC } \geq \mathbf{8}, \\
\mathbf{N}=\mathbf{1 0}\end{array}$ \\
\hline Strong & $24(11.2)$ & $9(22.0)$ & $2(20.0)$ \\
Medium & $19(8.9)$ & $0(0.0)$ & $5(50.0)^{\mathrm{a}}$ \\
Strong or medium & $43(20.1)$ & $9(22.0)$ & $7(70.0)^{\mathrm{b}}$ \\
Weak & $52(24.3)$ & $12(29.3)$ & $2(20.0)$ \\
All positive & $95(44.4)$ & $21(51.2)$ & $9(90.0)^{\mathrm{c}}$
\end{tabular}

a Medium: $M I C \geq 8$ vs. MIC $\leq 2, p=0.002$. ' Strong or medium: $M I C \geq 8$ vs. $M I C \leq 2, p=0.001$. ${ }^{c}$ Positive: $M I C \geq 8$ vs. $M I C \leq 2, p=0.007$. 
TABLE 7 | Relationship between biofilm-forming capacity and virulence factors.

\begin{tabular}{|c|c|c|c|c|c|c|}
\hline \multirow[t]{2}{*}{ Virulence factors (no. isolates tested) } & \multicolumn{5}{|c|}{ No. (\%) of isolates with biofilm phenotype } & \multirow[t]{2}{*}{$P^{a}$} \\
\hline & Strong & Medium & Strong or medium & Weak & All positive & \\
\hline esp+ (163) & $30(18.4)$ & $20(12.3)$ & $50(30.7)$ & $47(28.8)$ & $97(59.5)$ & $<0.001$ \\
\hline$e s p-(102)$ & $5(4.9)$ & $4(3.9)$ & $9(8.8)$ & $19(18.6)$ & $28(27.5)$ & \\
\hline gelE $+(171)$ & $12(7.0)$ & $13(7.6)$ & $25(14.6)$ & $46(26.9)$ & $71(41.5)$ & 0.013 \\
\hline gelE- $-(94)$ & $23(24.5)^{b}$ & $11(11.7)$ & $34(36.2)^{\mathrm{c}}$ & $20(21.3)$ & $54(57.4)$ & \\
\hline asa1+ (210) & $32(19.4)$ & $20(9.7)$ & $52(29.1)$ & $57(26.0)$ & $109(55.1)$ & 0.003 \\
\hline asa1- (55) & $3(5.5)$ & $4(7.3)$ & $7(12.7)$ & $9(16.4)$ & $16(29.1)$ & \\
\hline$c y \mid A+(178)$ & 26 (14.6) & 19 (10.7) & 45 (25.3) & 57 (32.0) & $102(57.3)$ & $<0.001$ \\
\hline cylA- (87) & $9(10.3)$ & $5(5.7)$ & $14(16.1)$ & $9(10.3)$ & $23(26.4)$ & \\
\hline$h y l+(83)$ & $7(8.4)$ & $6(7.2)$ & $13(15.7)$ & $20(24.1)$ & 33 (39.8) & 0.103 \\
\hline hyl- (182) & $28(15.4)$ & $18(9.9)$ & 46 (25.3) & 46 (25.3) & $92(50.5)$ & \\
\hline agg+ (88) & $11(12.5)$ & 12 (13.6) & $23(26.1)$ & $27(30.7)$ & $50(56.8)$ & 0.027 \\
\hline agg- (177) & $24(13.6)$ & $12(6.8)$ & 36 (20.3) & $39(22.0)$ & $75(42.4)$ & \\
\hline
\end{tabular}

a Biofilm formation: + vs. -. ' ${ }^{\mathrm{S} S t r o n g}$ biofilm: gelE-vs. gelE+, $p<0.001$. ${ }^{\mathrm{C}}$ Strong or medium biofilm: gelE-vs. gelE+, $p<0.001$.

\section{Virulence Factor Association with Biofilm Formation by Multiple Regression Analysis}

In order to determine the independent contribution of each virulence factor to the biofilm formation of E. faecalis, multiple logistic regression analysis was performed. Thus, esp, gelE, asal, $c y l A$, and $a g g$ were used as independent variables, and weak biofilm formation, strong or medium biofilm formation were used as a dependent variable in the multivariate model. The logistic regression model was constructed by a backward selection approach based on the Wald statistic. $P$-values $<0.05$ were regarded as statistically significant. As shown in Table 8, only one factor, $c y l A$, was found to be an independent risk factor for weak biofilm formation. Meanwhile, esp positivity was independently associated with strong or medium biofilm formation, and gelE emerged as an independent anti-risk factor for strong or medium biofilm formation.

\section{Expression Levels of the esp and cylA Genes in E. faecalis Clinical Isolates}

Last, in order to clarify the role of E. faecalis virulence factors during biofilm formation, the expression levels of the esp and cylA genes of E. faecalis clinical isolates were determined by qRT-PCR. For the detection of esp expression, eight clinical E. faecalis isolates that were esp positive, and with the same

TABLE 8 | Multivariate regression analysis of virulence factors associated with E. faecalis biofilm formation.

\begin{tabular}{lccr}
\hline Isolate biofilm phenotype & Factor & OR $(\mathbf{9 5 \%} \mathbf{C l})$ & $\boldsymbol{P}$ \\
\hline Weak biofilm formation & & & \\
& CyIA & $4.083(1.912-8.716)$ & $<0.001$ \\
Strong or medium biofilm formation & & & \\
& gelE & $0.300(0.159-0.564)$ & $<0.001$ \\
& cylA & $0.393(0.146-1.056)$ & 0.064 \\
& esp & $8.207(2.795-24.099)$ & $<0.001$
\end{tabular}

phenotypes of other virulence factors (asa1+; gelE-; cylA-; $h y l-; a g g+)$ were chosen. Among those 8 esp-positive isolates, the 16C383 strain with negative biofilm formation was set as the reference strain (esp expression $=1$ ). As shown in Figure 1, the esp expression was markedly elevated (11.39 to 134.08fold, respectively) in those strong biofilm isolates $(16 \mathrm{C} 1,16 \mathrm{C} 51$, $16 \mathrm{C} 126$, and 16C353), compared with the weak biofilm producers (16C29, 4.01-fold and 16C274, 3.47-fold). These results suggest that esp was only associated with strong biofilm, not with the weak biofilm formation of E. faecalis isolates, consistent with the previous findings.

For the test of $c y l A$ expression, nine clinical E. faecalis isolates that were $c y l A$ positive, and with the same phenotypes of other virulence factors ( $a s a 1+; g e l E-; e s p-; h y l-; a g g+)$ were chosen. Among those $9 \mathrm{cylA}$-positive isolates, the 16C281 strain with negative biofilm formation was set as the reference strain (cylA expression $=1$ ). As shown in Figure 2, the cylA gene expression was higher (4.02 to 6.00 -fold) in those weak biofilm isolates (16C24, 16C54, 16C305, 16C306, and 16C374), compared with in the negative biofilm isolate (16C394, 1.37-fold), or the medium (16C169, 2.82-fold) or strong (16C60, 1.70-fold) biofilm isolates. These data may confirm that the cylA gene was only associated with weak biofilm, not with the strong or medium biofilm formation of $E$. faecalis isolates.

\section{DISCUSSION}

Although E. faecalis is generally less drug resistant than E. faecium, we found that biofilm formation was more prevalent for E. faecalis than has been reported previously for E. faecium (Sandoe et al., 2003). The prevalence of E. faecalis biofilm observed in this study, $47.2 \%$, was lower than the $60-90 \%$ prevalence rates reported previously in Europe (Toledo-Arana et al., 2001; Dupre et al., 2003; Sandoe et al., 2003). In particular, the prevalence of $E$. faecalis biofilm in this study for isolates from urine, $50.4 \%$, and from blood, $40.0 \%$, were essentially half of the $100 \%$ prevalence rates reported previously for isolates from urine 


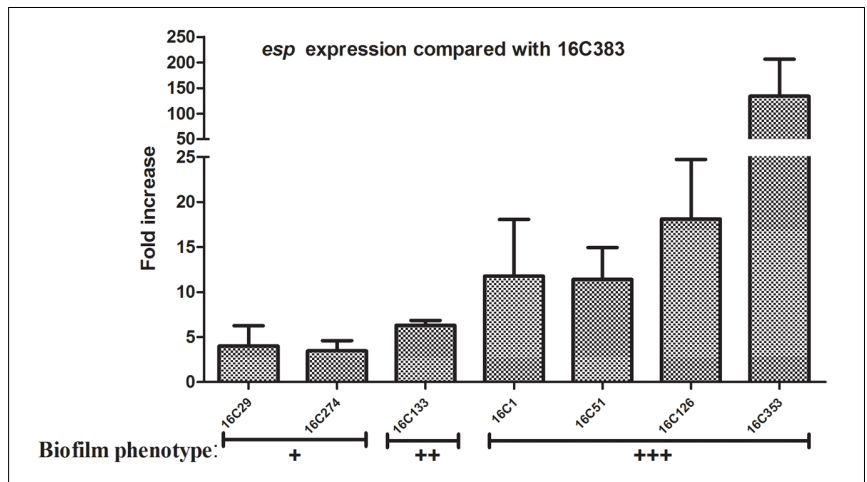

FIGURE 1 | Relative fold expression of esp in seven clinical Enterococcus faecalis isolates. The expression levels of esp were determined by quantitative real-time polymerase chain reaction (qRT-PCR), with the clinical $E$. faecalis isolate $16 \mathrm{C} 383$ as the reference strain (expression $=1$, biofilm negative). Biofilm phenotype: + , weak; ++, medium; +++, strong. The esp was overexpressed in the four strong biofilm strains (11.39 to 134.08-fold).

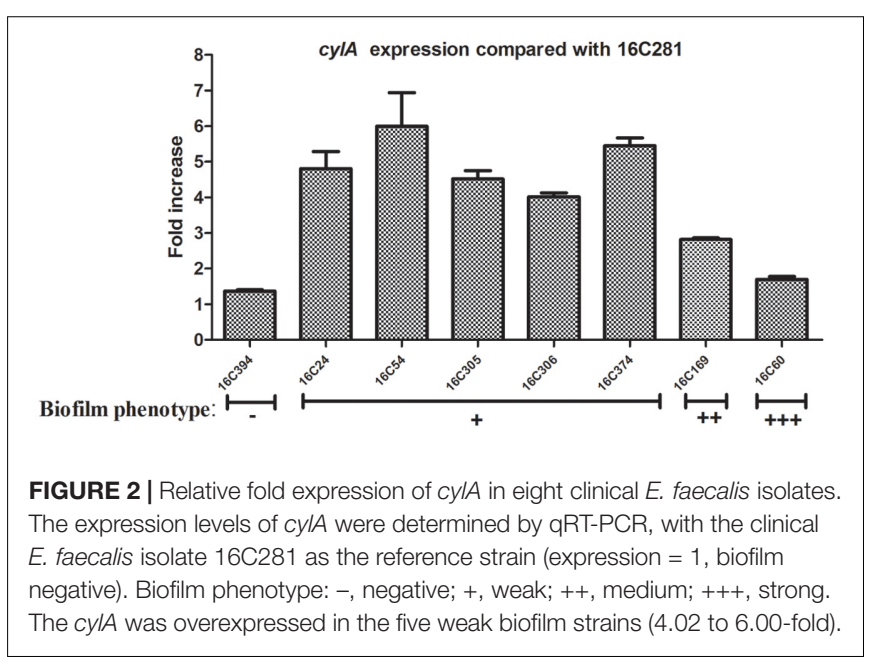

(Seno et al., 2005) and blood (Sandoe et al., 2003). Among the 57 E. faecalis isolates from urine with biofilm formation ability, $47.4 \%$ (27/57) of isolates were weak biofilm producers. Another study from Japan found in 352 E. faecalis strains from urinary tract infections that all of them had the capacity to form biofilm, $37.5 \%(132 / 352)$ of isolates exhibited weak biofilm formation (Seno et al., 2005). Our study found the cylA was associated with E. faecalis isolates with weak biofilm formation, and the prevalence of $c y l A$ in the biofilm-positive E. faecalis isolates from urine was $78.9 \%(45 / 57)$, which was higher than in the Seno et al. research $(46.6 \%, 164 / 352)$. Thus, the prevalence of the weak biofilm phenotype in those $E$. faecalis isolates from urine may be due to the expression of $c y l A$, based on the present study.

The lower rates of $E$. faecalis biofilm observed here could be due to several possible reasons. First, the E. faecalis strains isolated from different studies may have different ST frequencies. Second, because crystal violet staining analysis is a semiquantitative method, it carries a risk of operation error. Last, there is not yet a consistent criterion for the determination of a positive biofilm, particularly with respect to a crystal violet optical density cut-off value.

The present demonstration of 44 STs provides, to the best of our knowledge, the first MLST of E. faecalis in China. The dominance of ST16 and ST179 among our samples is somewhat consistent with a prior study in Malaysia, in which ST6, ST16, ST28, ST179, and ST399 were represented (Weng et al., 2013). Our finding of greater biofilm prevalence, especially strong/medium biofilm prevalence, among ST16 isolates than among ST179 isolates is noteworthy in light of previous evidence showing that ST16 isolates may be representative of hospitaladapted strains (Ruiz-Garbajosa et al., 2006), and the observation that, relative to planktonic bacteria, biofilms enable bacteria to better survive in adverse environmental conditions, including in the presence of antibiotics and disinfectants (Lebeaux et al., 2014). E. faecalis ST16 may, like S. epidermidis ST27 (Kozitskaya et al., 2005), occur preferentially in nosocomial environments, with an atypically high prevalence of biofilm formation relative to other STs of the microbe species.

The relationship between E. faecalis biofilm formation and antibiotic resistance had not been well explored before this study. Interest in VREs has been growing in recent years (O'Driscoll and Crank, 2015). Linezolid has been used to treat VRE infection, but wider use of linezolid may be increasing the prevalence of linezolid resistance in E. faecalis (Kainer et al., 2007). Indeed, here we found ten E. faecalis isolates that were resistant to linezolid, which was with no linezolid treatment, but found no VRE isolates. Moreover, we found that the prevalence of biofilm formation, especially the strong or medium biofilm formation, was significantly higher in linezolid-resistant E. faecalis isolates than in linezolid-sensitive isolates. Although E. faecalis ST16 was found to have a high prevalence of biofilm formation, ST16 isolates were similarly represented across linezolid-sensitive and -resistant strains, indicating that biofilm formation by linezolidresistant isolates cannot be attributed to the ST16 profile per se. Further research is needed to examine whether biofilm formation plays a role in E. faecalis resistance to linezolid.

The present finding that esp+ isolates are more likely to exhibit strong or medium biofilm formation than esp-isolates is consistent with several previous studies that have suggested a link between the virulence factor Esp and E. faecalis biofilm formation (Toledo-Arana et al., 2001; Tendolkar et al., 2004; Paganelli et al., 2012). Notwithstanding, other studies have failed to find evidence of such a link (Kristich et al., 2004; Mohamed and Murray, 2005; Di Rosa et al., 2006; Elhadidy and Elsayyad, 2013; Anderson et al., 2016). We did not observe an association between esp positivity and weak biofilm formation, suggesting the possibility that esp might enhance extant E. faecalis biofilm formation, without having a fundamental influence on the basic capacity for biofilm formation per se.

Our finding of gelE positivity being inversely associated with strong or medium biofilm formation in E. faecalis is surprising. Previous studies have suggested that the gelE-encoded protein, which can hydrolyze gelatin, collagen, and hemoglobin, may favor the development of E. faecalis biofilms (Kayaoglu and Ørstavik, 2004; Park et al., 2007; Thomas et al., 2008), while others found no significant correlation between the presence of gelE and 
biofilm formation in E. faecalis (Mohamed and Murray, 2005; Anderson et al., 2016). It might be that the STs of our E. faecalis strains differed from those of previous studies, or there may be as yet unidentified factors that determine E. faecalis biofilm formation.

Our negative finding with respect to asal affecting biofilm formation is inconsistent with a prior study showing as association between asal and E. faecalis biofilm formation (Chuang-Smith et al., 2010). Our finding that cylA was only a significant factor in weak biofilm formation suggests that $c y l A$ may be irrelevant to strong or medium biofilm formation in E. faecalis. The cylA gene has been reported previously to be associated with the E. faecalis biofilm formation of urinary tract isolates (Seno et al., 2005). However, thus far, there is little information regarding the potential role of $c y l A$ - which can lyse prokaryotic cells, as well as erythrocytes and other eukaryotic cells - in E. faecalis biofilm formation.

\section{CONCLUSION}

The present study showed that a large portion of E. faecalis isolates from clinical samples form biofilms readily. The MLST of E. faecalis in this study demonstrated substantial ST diversity, with the main STs being ST16 and ST179. In general, biofilm formation was more strongly associated with ST16 than ST179. Our findings suggest a positive association between linezolid resistance in E. faecalis and robust biofilm formation. Interestingly, we found that the cylA gene was associated with weak biofilm formation of E. faecalis isolates, while the esp gene was only associated with strong or medium biofilm formation of E. faecalis isolates.

\section{AUTHOR CONTRIBUTIONS}

$\mathrm{J}-\mathrm{XZ}$ participated in the design of the study, carried out the biofilm assay, analyzed and interpreted the data, and drafted the manuscript. YW participated in the analysis of the data

\section{REFERENCES}

Anderson, A. C., Jonas, D., Huber, I., Karygianni, L., Wölber, J., Hellwig, E., et al. (2016). Enterococcus faecalis from food, clinical specimens, and oral sites: prevalence of virulence factors in association with biofilm formation. Front. Microbiol. 6:1534. doi: 10.3389/fmicb.2015.01534

Bonten, M. J., and Willems, R. J. (2012). Vancomycin-resistant Enterococcuschronicle of a foretold problem. Ned. Tijdschr. Geneeskund. 156, A5233.

Chuang-Smith, O. N., Wells, C. L., Henry-Stanley, M. J., and Dunny, G. M. (2010). Acceleration of Enterococcus faecalis biofilm formation by aggregation substance expression in an ex vivo model of cardiac valve colonization. PLOS ONE 5:e15798. doi: 10.1371/journal.pone.0015798

Damborg, P., Top, J., Hendrickx, A. P., Dawson, S., Willems, R. J., and Guardabassi, L. (2009). Dogs are a reservoir of ampicillin-resistant Enterococcus faecium lineages associated with human infections. Appl. Environ. Microbiol. 75, 2360-2365. doi: 10.1128/AEM.02035-08

Di Rosa, R., Creti, R., Venditti, M., D’Amelio, R., Arciola, C. R., Montanaro, L. et al. (2006). Relationship between biofilm formation, the enterococcal surface protein (Esp) and gelatinase in clinical isolates of Enterococcus faecalis and and provided a critical revision of the manuscript. Z-WL conducted the RNA extraction, quantitative real-time PCR and the data analysis. Z-YP performed antibiotic-susceptibility testing, detected virulence genes by PCR, and participated in the data analysis. W-MY, ZC, and D-YL participated in the acquisition of the samples, isolated DNA, conducted MLST, and participated in the data analysis. Q-WD, DQ, and Z-JY designed the study, participated in the data analysis, and provided critical revisions of the manuscript for important intellectual content.

\section{FUNDING}

This work was supported by grants from the National Natural Science Foundation of China (no. 81170370), from a grant from Sanming Project of Medicine in Shenzhen (SMGC201705029), the Shenzhen Scientific Research Program (Nos. JCYJ20150402152130167, JCYJ20150402152130173, and JCYJ20170307153714512), the Scientific Research Project of the Shenzhen Health and Family Planning System (no. 201601058), and the Shenzhen Nanshan District Scientific Research Program of China (no. 2016010).

\section{ACKNOWLEDGMENTS}

The authors would like to thank Xiao-fei Wang and Zhi-hui Lv (Key Laboratory of Medical Molecular Virology of Ministries of Education and Health, School of Basic Medical Science and Institutes of Biomedical Sciences, Shanghai Medical College of Fudan University) for their excellent technical support and suggestions.

\section{SUPPLEMENTARY MATERIAL}

The Supplementary Material for this article can be found online at: https://www.frontiersin.org/articles/10.3389/fmicb. 2017.02338/full\#supplementary-material

Enterococcus faecium. FEMS Microbiol. Lett. 256, 145-150. doi: 10.1111/j.15746968.2006.00112.x

Dupre, I., Zanetti, S., Schito, A. M., Fadda, G., and Sechi, L. A. (2003). Incidence of virulence determinants in clinical Enterococcus faecium and Enterococcus faecalis isolates collected in Sardinia (Italy). J. Med. Microbiol. 52, 491-498. doi: 10.1099/jmm.0.05038-0

Elhadidy, M., and Elsayyad, A. (2013). Uncommitted role of enterococcal surface protein, Esp, and origin of isolates on biofilm production by Enterococcus faecalis isolated from bovine mastitis. J. Microbiol. Immunol. Infect. 46, 80-84. doi: 10.1016/j.jmii.2012.02.002

Fisher, K., and Phillips, C. (2009). The ecology, epidemiology and virulence of Enterococcus. Microbiology 155, 1749-1757. doi: 10.1099/mic.0.026385-0

Flokas, M. E., Karageorgos, S. A., Detsis, M., Alevizakos, M., and Mylonakis, E. (2017). Vancomycin-resistant enterococci colonisation, risk factors and risk for infection among hospitalised paediatric patients: a systematic review and metaanalysis. Int. J. Antimicrob. Agents 49, 565-572. doi: 10.1016/j.ijantimicag.2017. 01.008

Gawryszewska, I., Żabicka, D., Hryniewicz, W., and Sadowy, E. (2017). Linezolidresistant enterococci in Polish hospitals: species, clonality and determinants 
of linezolid resistance. Eur. J. Clin. Microbiol. Infect. Dis. 36, 1279-1286. doi: 10.1007/s10096-017-2934-7

Kainer, M. A., Devasia, R. A., Jones, T. F., Simmons, B. P., Melton, K., Chow, S., et al. (2007). Response to emerging infection leading to outbreak of linezolidresistant enterococci. Emerg. Infect. Dis. 13, 1024-1030. doi: 10.3201/eid1307. 070019

Kayaoglu, G., and Ørstavik, D. (2004). Virulence factors of Enterococcus faecalis: relationship to endodontic disease. Crit. Rev. Oral Biol. Med. 15, 308-320. doi: $10.1177 / 154411130401500506$

Kozitskaya, S., Olson, M. E., Fey, P. D., Witte, W., Ohlse, K., and Ziebuhr, W. (2005). Clonal analysis of Staphylococcus epidermidis isolates carrying or lacking biofilm-mediating genes by multilocus sequence typing. J. Clin. Microbiol. 43, 4751-4757. doi: 10.1128/JCM.43.9.4751-4757.2005

Kristich, C. J., Li, Y. H., Cvitkovitch, D. G., and Dunny, G. M. (2004). Esp independent biofilm formation by Enterococcus faecalis. J. Bacteriol. 186, 154-163. doi: 10.1128/JB.186.1.154-163.2004

Lebeaux, D., Ghigo, J. M., and Beloina, C. (2014). Biofilm-related infections: bridging the gap between clinical management and fundamental aspects of recalcitrance toward antibiotics. Microbiol. Mol. Biol. Rev. 78, 510-543. doi: 10.1128/MMBR.00013-14

Manning, S. D., Springman, A. C., Lehotzky, E., Lewis, M. A., Whittam, T. S., and Davies, H. D. (2009). Multilocus sequence types associated with neonatal group B streptococcal sepsis and meningitis in Canada. J. Clin. Microbiol. 47, 1143-1148. doi: 10.1128/JCM.01424-08

Mohamed, J. A., Huang, W., Nallapareddy, S. R., Teng, F., and Murray, B. E. (2004). Influence of origin of isolates, especially endocarditis isolates, and various genes on biofilm formation by Enterococcus faecalis. Infect. Immun. 72, 3658-3663. doi: 10.1128/IAI.72.6.3658-3663.2004

Mohamed, J. A., and Murray, B. E. (2005). Lack of correlation of gelatinase production and biofilm formation in a large collection of Enterococcus faecalis isolates. J. Clin. Microbiol. 43, 5405-5407. doi: 10.1128/JCM.43.10.5405-5407. 2005

Montealegre, M. C., La Rosa, S. L., Roh, J. H., Harvey, B. R., and Murray, B. E. (2015). The Enterococcus faecalis EbpA Pilus protein: attenuation of expression, biofilm formation, and adherence to fibrinogen start with the rare initiation codon ATT. mBio. 6:e00467-15. doi: 10.1128/mBio.00467-15

O'Driscoll, T., and Crank, C. W. (2015). Vancomycin-resistant enterococcal infections: epidemiology, clinical manifestations, and optimal management. Infect. Drug Resist. 8, 217-230. doi: 10.2147/IDR.S54125

Paganelli, F. L., Willems, R. J., and Leavis, H. L. (2012). Optimizing future treatment of enterococcal infections: attacking the biofilm? Trends Microbiol. 20, 40-49. doi: 10.1016/j.tim.2011.11.001

Park, S. Y., Kim, K. M., Lee, J. H., Seo, S. J., and Lee, I. H. (2007). Extracellular gelatinase of Enterococcus faecalis destroys a defense system in insect hemolymph and human serum. Infect. Immun. 75, 1861-1869. doi: 10.1128/IAI.01473-06

Parker, R. E., Laut, C., Gaddy, J. A., Zadoks, R., Davies, H. D., and Manning, S. (2016). Association between genotypic diversity and biofilm production in group B Streptococcus. BMC Microbiol. 16:86. doi: 10.1186/s12866-016-0704-9

Ruiz-Cruz, S., Espinosa, M., Goldmann, O., and Bravo, A. (2015). Global regulation of gene expression by the MafR protein of Enterococcus faecalis. Front. Microbiol. 6:1521. doi: 10.3389/fmicb.2015.01521

Ruiz-Garbajosa, P., Bonten, M., Robinson, D., Robinson, A., Top, J., Nallapareddy, S., et al. (2006). Multilocus sequence typing scheme for Enterococcus faecalis reveals hospital-adapted genetic complexes in a background of high rates of recombination. J. Clin. Microbiol. 44, 2220-2228. doi: 10.1128/JCM.02596-05

Sandoe, J. A., Witherden, I. R., Cove, J. H., Heritage, J., and Wilcox, M. H. (2003). Correlation between enterococcal biofilm formation in vitro and medicaldevice-related infection potential in vivo. J. Med. Microbiol. 52, 547-550. doi: 10.1099/jmm.0.05201-0
Seno, Y., Kariyama, R., Mitsuhata, R., Monden, K., and Kumon, H. (2005). Clinical implications of biofilm formation by Enterococcus faecalis in the urinary tract. Acta Med. Okayama 59, 79-87.

Sghir, A., Gramet, G., Suau, A., Rochet, V., Pochart, P., and Dore, J. (2000). Quantification of bacterial groups within human fecal flora by oligonucleotide probe hybridization. Appl. Environ. Microbiol. 66, 2263-2266. doi: 10.1128/ AEM.66.5.2263-2266.2000

Süßmuth, S. D., Muscholl-Silberhorn, A., Wirth, R., Susa, M., Marre, R., and Rozdzinski, E. (2000). Aggregation substance promotes adherence, phagocytosis, and intracellular survival of Enterococcus faecalis within human macrophages and suppresses respiratory burst. Infect. Immunity 68, 4900-4906. doi: 10.1128/iai.68.9.4900-4906.2000

Tendolkar, P. M., Baghdayan, A. S., Gilmore, M. S., and Shankar, N. (2004). Enterococcal surface protein, Esp, enhances biofilm formation by Enterococcus faecalis. Infect. Immun. 72, 6032-6039. doi: 10.1128/IAI.72.10.6032-6039.2004

Thomas, V. C., Thurlow, L. R., Boyle, D., and Hancock, L. E. (2008). Regulation of autolysis-dependent extracellular DNA release by Enterococcus faecalis extracellular proteases influences biofilm development. J. Bacteriol. 190, 5690-5698. doi: 10.1128/JB.00314-08

Toledo-Arana, A., Valle, J., Solano, C., Arrizubieta, M., Cucarella, C., Lamata, M., et al. (2001). The enterococcal surface protein, Esp, is involved in Enterococcus faecalis biofilm formation. Appl. Environ. Microbiol. 67, 4538-4545. doi: 10.1128/AEM.67.10.4538-4545.2001

Treitman, A. N., Yarnold, P. R., Warren, J., and Noskin, G. A. (2005). Emerging incidence of Enterococcus faecium among hospital isolates (1993 to 2002). J. Clin. Microbiol. 43, 462-463. doi: 10.1128/JCM.43.1.462-463.2005

Urwin, R., and Maiden, M. C. (2003). Multi-locus sequence typing: a tool for global epidemiology. Trends Microbiol. 11, 479-487. doi: 10.1016/j.tim.2003.08.006

Van Harten, R. M., Willems, R. J. L., Martin, N. I., and Hendrickx, A. P. A. (2017). Multidrug-resistant enterococcal infections: new compounds, novel antimicrobial therapies? Trends Microbiol. 25, 467-479. doi: 10.1016/j.tim.2017. 01.004

Van Tyne, D., Martin, M. J., and Gilmore, M. S. (2013). Structure, function, and biology of the Enterococcus faecalis cytolysin. Toxins 5, 895-911. doi: 10.3390/ toxins5050895

Vankerckhoven, V., Autgaerden, T., Vael, C., Lammens, C., Chapelle, S., Rossi, R., et al. (2004). Development of a multiplex PCR for the detection of asa1, gelE, cylA, esp, and hyl genes in enterococci and survey for virulence determinants among European hospital isolates of Enterococcus faecium. J. Clin. Microbiol. 42, 4473-4479. doi: 10.1128/JCM.42.10.4473-4479.2004

Weng, P. L., Ramli, R., Shamsudin, M. N., Cheah, Y. K., and Hamat, R. A. (2013). High genetic diversity of Enterococcus faecium and Enterococcus faecalis clinical isolates by pulsed-field gel electrophoresis and multilocus sequence typing from a hospital in Malaysia. Biomed Res. Int. 2013:938937. doi: 10.1155/2013/ 938937

Xu, T., Wu, Y., Lin, Z. W., Bertram, R., Götz, F., Zhang, Y., et al. (2017). Identification of genes controlled by the essential YycFG two-component system reveals a role for biofilm modulation in Staphylococcus epidermidis. Front. Microbiol. 8:724. doi: 10.3389/fmicb.2017.00724

Conflict of Interest Statement: The authors declare that the research was conducted in the absence of any commercial or financial relationships that could be construed as a potential conflict of interest.

Copyright (c) 2017 Zheng, Wu, Lin, Pu, Yao, Chen, Li, Deng, Qu and Yu. This is an open-access article distributed under the terms of the Creative Commons Attribution License (CC BY). The use, distribution or reproduction in other forums is permitted, provided the original author(s) or licensor are credited and that the original publication in this journal is cited, in accordance with accepted academic practice. No use, distribution or reproduction is permitted which does not comply with these terms. 